\title{
A review on intelligent reflecting surface-based terahertz communication
}

\author{
Arpita Patel ${ }^{1}$, Aasheesh Shukla ${ }^{2}$ \\ ${ }^{1}$ Department of Electronics and Communication, Faculty of Technology and Engineering, CHARUSAT University, Gujarat, India \\ ${ }^{2}$ Department of Electronics and Communication, Faculty of Technology and Engineering, GLA University, Mathura, Gujarat, India
}

\section{Article Info \\ Article history: \\ Received Oct 17, 2021 \\ Revised Dec 22, 2021 \\ Accepted Jan 6, 2022}

Keywords:

$6 \mathrm{G}$

Coverage capability

Intelligent reflecting surface

Signal coverage

Terahertz communication

\begin{abstract}
Terahertz (THz)-band communications can be considered as the new frontline for future-generation wireless communication systems as it has a wider bandwidth in comparison to microwave and mmwave communications. It also promises to integrate the wide range of diverse applications. Because of high path loss and narrow spreading of $\mathrm{THz}$ waves, the transmission is extremely sensitive and signal coverage is a critical problem. To overcome this, an intelligent reflecting surface (IRS), has received great interest which steers the beams of $\mathrm{THz}$ waves by regulating the phase shifts to alleviate blockage and improve the coverage capability. In this paper, the role of IRS in conjunction with the $\mathrm{THz}$ communication has been discussed. Further, challenges and research directions of IRS for terahertz communication are also discussed.
\end{abstract}

This is an open access article under the CC BY-SA license.

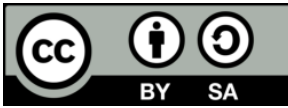

\section{Corresponding Author:}

Arpita Patel

Department of Electronics and Communication, Faculty of Technology and Engineering

CHARUSAT University

Nadiad-Petlad Rd, Highway, Changa, Gujarat 388421, India

Email: arpitapatel.ec@ charusat.ac.in

\section{INTRODUCTION}

Sixth generation $(6 \mathrm{G})$ wireless communication networks have attracted more attention to support the numerous applications in the near future. Figure 1 presents an $6 \mathrm{G}$ system overview along with some enabling technologies and use cases. In the case of the telecommunication sector, the race is towards improving the data rate to fulfill different applications like virtual reality (VR), wireless data center, on-chip communication [1], [2]. This never ending quest of high data rate and the growth of wireless usage sets the target for the research community to discover suitable regions in the radio spectrum to satisfy the growing needs of individuals. Following this trend, wireless terahertz $(\mathrm{THz})$ band are getting noticeable attention within the global community that can resolve the spectrum shortage problem and can enhance the capacity of wireless system [3]. The THz band is the band that spans from 0.1 to $10 \mathrm{THz}$. There are several reasons that motivates the use of THz band like seamless data transfer, unlimited bandwidth, low latency and high download rate.

A new horizon in the field of communications deployed for $5 \mathrm{G}$ technology by many funding agenesis who supports the $\mathrm{THz}$ projects. The list of the latest terahertz projects [4]. THz waves are alluring for remote interchanges since they can offer tremendous transfer speed and large bandwidth, which is fundamental for expanding data capacity. Nonetheless, there is an undeniable disadvantage: large signal loss. The attenuation in the atmosphere at frequencies over $100 \mathrm{GHz}$ is a lot bigger than that in the microwave recurrence band and the huge loss not just limits the service yet additionally corrupts the signal to noise (SNR) of the system, which impacts data capacity as well. 
Several studies reviewed and discribed the limitations and potential benefits of the $\mathrm{THz}$ band. The first survey was on $\mathrm{THz}$ applications, sources and sensors for frequencies above $500 \mathrm{GHz}$. After that an article have been published for terahertz sources, detector and modulators for various applications [5]. Federici and Moeller [6], Channel modeling for THz communication with some investigation results are presented. In 2010, a review on basic channel modeling, implementation and detection issues for $\mathrm{THz}$ bands are preseted [7]. A study on $\mathrm{THz}$ modulators, channel modeling and research directions for future wireless communication systems is studied [8]. Huang and Wang [9], shed light on THz communication for short range network as substitute for high data rate communication Systems. Nagatsuma [10], an overview of current advances in $\mathrm{THz}$ generation with phonetics is presented,which can achieve data rate up to $100 \mathrm{Gbps}$ on real time or offline. Akyildiz et al. [2], the challenges and applications in generation of the THz band has discussed. Kürner and Priebe [11], have demonstrated some research projects and spectrum regulations. Hirata and Yaita [12], the challenges and opportunities in THz communications for vehicular networks is presented. Sengupta et al. [13], review of research, development and implementation test beds in generating $\mathrm{THz}$ signals for communication, sensing and imaging applications is discussed. The process in wave propagation and channel modeling for wireless on chip communication from $\mathrm{mm}$ wave to $\mathrm{THz}$ and optics are presented [14], [15]. Han et al. [3], provides a brief review on applications for $\mathrm{THz}$ band and research directions of $6 \mathrm{G}$ Heterogeneous network. $\mathrm{THz}$ channel feature and current advancement in device technologies are reviewed [1]. Comprehensive comparison amongst the $\mathrm{THz}$ communication and its other contenders of $\mathrm{THz}$ communication are reviewed with its potential use cases of $\mathrm{THz}$ band [16]. Recently a survey on comparison of the type and materials for THz antennas and the issues, challenges and opportunities are presented [17]. Rahaman et al. [18], attributes and issues of $\mathrm{THz}$ range with some test-case scenario are presented. An overview of recent progresses in signal processing techniques for THz communications is presented [19]. Finally, Sarieddeen et al. [20], applications of imaging, sensing, and localization at the $\mathrm{THz}$ band with proof f-concept simulations are presented. The aforementioned review articles are itemized in Table 1 focus on review for related technologies for $\mathrm{THz}$ Band.

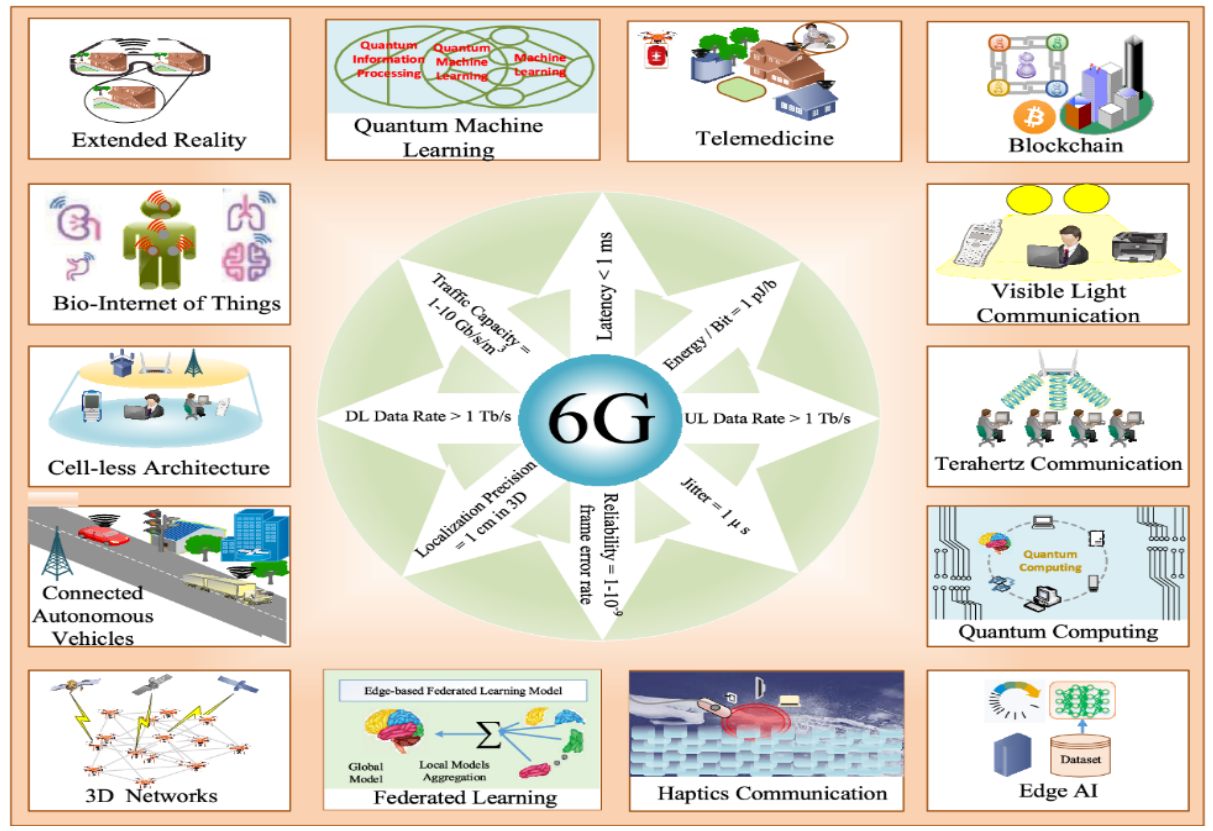

Figure 1.6G system overview [21]

At present, there is still a demand to have a complete vision on the existing development and new improvements in the field of $\mathrm{THz}$ communication which can help scientists to draw revolutionary footsteps for several communication systems. By contrast, this is the first reveiw on intelligent reflecting surface (IRS) added $\mathrm{THz}$ communication systems which also presents the open research challenges and future research directions. Further, the paper is organized as follows: in section 2, introduction to IRS and terahertz communication is presented. Section 3 categorize the recent studies of intelligent reflecting surfaces for THz. In section 4, some directions for forthcoming research of the porposed work have presented and finally, section 5 concludes the paper. 
Table 1. Brief review on $\mathrm{THz}$ communication

\begin{tabular}{|c|c|c|}
\hline Year & Focus & Ref \\
\hline 2002 & $\begin{array}{l}\text { This paper is the first survey on sources, sensors and application for terahertz with } \\
\text { frequency higher than } 500 \mathrm{GHz}-10\end{array}$ & {$[22]$} \\
\hline 2004 & A review on terahertz sources, detector and modulators for various applications & [5] \\
\hline 2009 & Investigation results for channel modeling in terahertz communication are presented & [6] \\
\hline 2010 & $\begin{array}{l}\text { Basic channel modeling techniques, generation methods, detection methods and } \\
\text { antennas for } \mathrm{THz} \text { communication is presented with some link measurements. }\end{array}$ & [7] \\
\hline 2011 & $\begin{array}{l}\text { A review on } \mathrm{THz} \text { technologies, channel modeling with research directions for future } \\
\text { wireless communication systems is presented }\end{array}$ & [8] \\
\hline 2011 & $\begin{array}{l}\text { This paper surveys short range network for } \mathrm{THz} \text { communication as a substitute for } \\
\text { high data rate as forthcoming wireless communication systems }\end{array}$ & [23] \\
\hline 2011 & The development in THz technology with its applications are reviewed & [9] \\
\hline 2013 & $\begin{array}{l}\text { A paper presents survey on current developments in } \mathrm{THz} \text { technology using } \\
\text { phonetics to achieve data rate upto } 100 \mathrm{Gbps}\end{array}$ & {$[10]$} \\
\hline 2014 & $\begin{array}{l}\text { This paper presents review on } \mathrm{THz} \text { applications, challenges and channel modeling } \\
\text { with some experimental and simulation testbeds. }\end{array}$ & {$[2]$} \\
\hline 2014 & $\begin{array}{l}\text { A survey on research projects, regulations and standardization for } \mathrm{THz} \\
\text { communications is presented }\end{array}$ & [11] \\
\hline 2015 & $\begin{array}{l}\text { A review on research for } \mathrm{THz} \text { communication along with its development and } \\
\text { implementation is presented }\end{array}$ & {$[12]$} \\
\hline 2016 & $\begin{array}{l}\text { A review on wave propagation and channel modeling for wireless in chip scale } \\
\text { wireless communication from mm wave to } \mathrm{THz} \text { and optics }\end{array}$ & [24] \\
\hline 2017 & $\begin{array}{l}\text { Review on terahertz band opportunities and challenges for vehicular networks is } \\
\text { presented }\end{array}$ & [14] \\
\hline 2018 & $\begin{array}{l}\text { A brief review on progress of } \mathrm{THz} \text { integrated electronic and electronic-photonic } \\
\text { systems is presented }\end{array}$ & [13] \\
\hline 2019 & $\begin{array}{l}\text { This article provides a brief review on } \mathrm{THz} \text { frequency assisted wireless systems, } \\
\text { applications of utilizing } \mathrm{THz} \text { bands and the research directions of an ultra-fast } \\
\text { beyond } 5 \mathrm{~g} \text { heterogeneous network }\end{array}$ & [3] \\
\hline 2019 & $\begin{array}{l}\text { A review on the progress to } \mathrm{THz} \text { communications with few key enabling } \\
\text { technologies for } \mathrm{THz} \text { systems are presented }\end{array}$ & [1] \\
\hline 2019 & $\begin{array}{l}\text { The article provides THz frequency generation and channel models along with } \\
\text { future research direction }\end{array}$ & [16] \\
\hline 2020 & $\begin{array}{l}\text { This article discusses and compares various parameters like type and materials for } \\
\mathrm{THz} \text { antennas and with the issues, challenges and for the THz antennas }\end{array}$ & {$[17]$} \\
\hline 2020 & This paper provides the technology roadmap for $\mathrm{THz}$ communication & [18] \\
\hline 2020 & $\begin{array}{l}\text { An overview of recent progresses in signal processing techniques for } \mathrm{THz} \\
\text { communications }\end{array}$ & [19] \\
\hline 2020 & $\begin{array}{l}\text { Reviewed the applications of imaging, sensing, and localization at the } \mathrm{THz} \text { band } \\
\text { with proof-of-concept simulations }\end{array}$ & {$[20]$} \\
\hline
\end{tabular}

\section{INTELLIGENT REFLECTING SURFACES AND THZ COMMUNICATION}

\subsection{THz communication}

The explosion of wireless device and services have raised the demand to satisfy the requirement for high data rate transmission. The technology like millimeter wave ( $\mathrm{mm}$ wave-30-300 $\mathrm{GHz}$ ), massive multipleinput multiple-output (MIMO), beamforming has already considered as enablers of fifth-generation (5G) of wireless mobile communications. While due to trend of super high data rate and low latency requirements, it is difficult to handle the quality of service in $\mathrm{mm}$ wave systems for $5 \mathrm{G}$. To remove these barriers in networks beyond $5 \mathrm{G}$, one need to explore the resource $\mathrm{s}$ and technologies to validate. $\mathrm{THz}$ technology is one of those technologies which are expected to become main trend and shall play important role in beyond 5G (i.e., 6G) communication systems. THz band is the last unexplored piece of the spectrum and it is sandwich between microwave and optical band. Hence one can explore the technologies from both sides to support $\mathrm{THz}$ communications. Being a neighbor to $\mathrm{mm}$ wave spectrum, $\mathrm{THz}$ band $(0.1$ to $10 \mathrm{THz})$ has potential to fulfill the future demands of $6 \mathrm{G}$ wireless communication systems. The user in remote areas is difficult to access and needs a high data rate up to $10 \mathrm{Gbits} / \mathrm{s}$ per user. $\mathrm{THz}$ transmission will be an important block to face this challenge and can give high speed internet access everywhere.

\subsection{Intelligent reflecting surfaces (IRS)}

The $6 \mathrm{G}$ network needs more strict requirements than $5 \mathrm{G}$, like higher data rate, global coverage and connectivity and low latency. These cannot be fulfilled with existing technology for $5 \mathrm{G}$ services, which need to deploy more base stations and antennas to achieve enhance network coverage and capacity. Also, it requires to migrate to $\mathrm{mm}$ wave and even terahertz frequency to use large bandwidth to compensate for prorogation loss. By looking at above issues, it is necessary to develop new and innovative technologies to have more supportable capacity with low cost and complexity for future wireless networks. IRS, is a recently new paradigm for wireless communication systems. An IRS is a planar surface with an array of passive reflecting 
elements. These elements can independently impose the required phase shift on the incoming signal [25], [26]. The developments in metamaterials [27] can lead to the recofiguration of reflection coefficient in the real time to adapt the fulatuating wireless enviornment. IRS is also very benificial for practical implementation. It has a very low-cost hardware due to low cost printed dipole which can reflect without any radio frequency (RF) chains. Also, it is free form antenna noise and interference and can be easily mounted on object as it is having a light weight. Due to all this advantages, IRS is applicable in wireless network at terahertz frequency to enhance its spectral efficiency with low cost. In summary we can argue that, to compensate for high propagation loss at terahertz frequency, IRS is a good choice. Though, there is a limited paper to incorporate Intellignet surfaces into $\mathrm{THz}$ communication. The next section discusses the classification of IRS for THz.

\section{PROPOSED STUDIES OF INTELLIGENT REFLECTING SURFACES USE IN THZ BAND}

In this section the recent research work on IRS in THz communication have been classified. For IRS based communications systems, meta-materials and metasurface based design, power/spectral optimizations, channel estimation and sum rate analysis for IRS assisted THz. We also review use of IRSs in security-based communication systems, and other novel applications.

\subsection{Channel estimation in IRS based communications}

A novel method of estimating the average channel capacity per user of a spread spectrum MIMO multiple-user system is discussed [28], [29]. Since IRS has no capacity to transmitting and receiving the signals, the channel state information (CSI) of IRS-based system is isolated through old channel estimation methods. So, to obtain the complete CSI for IRS driven systems is the main problem. To overcome this challenge, numerous works have proposed so as to look into novel channel estimation methods for the IRS-assisted THz communications.

A hybrid beam forming architectures for channel estimation along with transmission solutions for IRS assisted system with massive MIMO in THz communication is presented [30]. They invoked beam training method for channel measurement. The analysis of beam pattern and quantization error are also assessed. A search code book design is proposed toreduce the complecity in channel estinamtion. Then, precoder/combiner and IRS designs and cooperative channel estimation method is also proposed which achieves a good spectral efficiency compared to non-IRS assisted approach. Chen et al. [31] have used the graphene-based IRS and developed a low complexity channel estimation scheme by utilizing the sparsity characteristics of $\mathrm{THz}$ channel. Wang et al. [32] addresses channel estimation problem using deep learning perspective for enabled MIMO system in THz. They have used sparse nature of $\mathrm{THz}$ channel and developed low complexity channel estimation scheme. Channel estimation issue is tackled with cooperative beam training scheme without searching exact path for departure/arrival [33]. They have concluded that only fewer angel differences need to search with fewer code words. The authors have first designed ternary-tree search at base station (BS) with the use of two novel training codebooks which balance between noise and complexity. Then hybrid beamforming methods are proposed to maximize the achievable rate. Chen et al. [34], the channel estimation and data rate maximization for IRS-aided THz MIMO system for 6G indoor applications is discussed further. Table 2 focus on review on channel estimation in THz band using IRS.

Table 2. Using IRS for channel estimation in $\mathrm{THz}$

\begin{tabular}{|c|c|c|c|c|}
\hline Ref & Scheme & Design methodology & Application scenario & Justification \\
\hline [30] & $\begin{array}{l}\text { Massive } \\
\text { MIMO }\end{array}$ & $\begin{array}{l}\text { Cooperative channel measurement via } \\
\text { beam training and codebook }\end{array}$ & THz communications & Improved spectral efficiency \\
\hline [31] & MIMO & $\begin{array}{l}\text { Compressed sensing channel estimation } \\
\text { with graphene IRS }\end{array}$ & 6G indoor application & $\begin{array}{l}\text { Consistent channel recovery performance } \\
\text { with extreme complexity reduction }\end{array}$ \\
\hline [32] & MIMO & $\begin{array}{l}\text { Channel estimation using deep learning } \\
\text { with the use of sparse channel } \\
\text { configuration }\end{array}$ & THz communications & $\begin{array}{l}\text { Better recovery performance with } \\
\text { reduced computational complexity }\end{array}$ \\
\hline [33] & $\begin{array}{l}\text { Massive } \\
\text { MIMO }\end{array}$ & $\begin{array}{l}\text { Cooperative channel estimation via joint } \\
\text { beam training and hybrid beam forming }\end{array}$ & THz communications & $\begin{array}{l}\text { Improvement in the achievable data rate } \\
\text { under the estimated CSI }\end{array}$ \\
\hline [34] & MIMO & $\begin{array}{l}\text { Joint channel estimation and data rate } \\
\text { maximization }\end{array}$ & Indoor $6 \mathrm{G}$ application & $\begin{array}{l}\text { Improved communication rate with } \\
\text { reduced computational complexity }\end{array}$ \\
\hline
\end{tabular}

\subsection{IRS for Secure communications in $\mathrm{THz}$ band}

Intelligent reflecting surface has gained much attention in secure transmission to reduce the path loss in THz band. IRS wisely adjusts the phase shifts so as to reduce the information leakage and signal power is navigated to desired user. We now see that how IRS can be used in THz band for secure transmission. Qiao and Alouini [35] have focused on secure transmission via IRS for mm wave and terahertz system. The security 
rate of this system is optimized by the jointinty optimizing the beam forming at base station and discrete phase shift at IRS. Ning et al. [36] uses active beam former and passive phase shifter at the base station and at IRS respectively for maximizing the secrecy rate. To tackle the non convex problem, a joint design is proposed to optimize the phase shifter and beam former. Chen et al. [37], security rate of the IRS based multi-inputs singleoutput (MISO) system is exploited with the use of discrete phase-shifts method. An overview of secure transmission in tera herts band using IRS is discussed in Table 3.

Table 3. Using IRS for secure transmission in $\mathrm{THz}$

\begin{tabular}{cclll}
\hline Ref & Scheme & \multicolumn{1}{c}{ Design methodology } & \multicolumn{1}{c}{ Application scenario } & Justification \\
\hline$[35]$ & $\begin{array}{c}\text { IRS-based THz } \\
\text { system }\end{array}$ & $\begin{array}{l}\text { Joint optimization of transmit beam } \\
\text { forming and reflecting matrix } \\
\text { Active beamforming and the passive } \\
\text { reflecting phase shifters }\end{array}$ & $\begin{array}{l}\text { Mm wave and THz } \\
\text { communications } \\
\text { THz communications }\end{array}$ & $\begin{array}{l}\text { Improvement in secrecy rate } \\
\text { with low complexity }\end{array}$ \\
[36] & MISO & Discrete phase-shift and precoder & THz communications & Improved secrecy rate \\
\hline
\end{tabular}

\subsection{Data rate aided IRS for $\mathrm{THz}$ communication}

An IRS is a collection of passive reflecting elements, which individually execute the required phase shift on the received signal. The sum rate for IRS based $\mathrm{THz}$ communication is exploited by improving the phase shift at IRS. The data rate of IRS based THz MIMO system is improved [34] by using joint approach of data rate and channel estimation method. Here, the channel estimation is performed by iterative atom pruning based subspace pursuit scheme and with the help of this estimated CSI, various data rate maximization techniques are suggested for the hunt of phase shifts of IRS elements. Amongst them, deep neural networkbased method gives optimum data rate performance by lower computational complexity. Shen et al. [38], two methods have been proposed to maximize the data rate performance by choosing best phase shift of IRS. A recent work by [39] examines that to improve the data rate of $\mathrm{THz}$ transmission using IRS, it is imperious to optimize the reflecting phase shift and sub-band allocation simuntaneously. Graphene-based IRS technique to increase coverage performance in indoor $\mathrm{THz}$ communication is proposed [40]. Here the, coverage problem is first converted into discrete phase shift problem and then, a novel suboptimal phase shift search technique is suggested which greatly reduce the complexity of exhaustive search method and also improve the coverage performance. The trajectory of unmanned aerial vehicle (UAV), phase shift of IRS with THz sub bands and power control is examined by [41] to achieve the higher data rate. Hao et al. [42], the joint analysis of hybrid beam forming and reflection matrix is examined to increase the data rate at BS and IRS respectively. Authors have considered imperfect CSI from IRS to users and jointly optimized the algorithm again and proved that channel estimation is playing important role on system data rate. Analytical physical model to enhance communication distance and data-rate for ultra-massive multiple-input multiple-output (UM-MIMO) at mm and $\mathrm{THz}$ band frequencies is proposed [43]. In their approach they have used plasmonic antenna arrays for transmission, reception and waveguiding. Table 4 shows various schemes of sum rate maximization in IRS for $\mathrm{THz}$ communication as mentined above.

Table 4. Sum rate maximization in IRS aided $\mathrm{THz}$ communication

\begin{tabular}{|c|c|c|c|c|}
\hline Ref & Scheme & Design methodology & Application scenario & Justification \\
\hline [29] & MIMO & $\begin{array}{l}\text { Joint approach of channel estimation sum } \\
\text { rate maximization }\end{array}$ & $\begin{array}{l}\text { Indoor } \mathrm{THz} \\
\text { communications }\end{array}$ & $\begin{array}{l}\text { Improved communication rate with reduced } \\
\text { computational complexity }\end{array}$ \\
\hline$[38]$ & MIMO & $\begin{array}{l}\text { Optimization of phase shift of reflecting } \\
\text { surface }\end{array}$ & THz communications & Improved the sum-rate \\
\hline [39] & & $\begin{array}{l}\text { Location of IRS, phase shift, sub-band } \\
\text { allocation, and power control for UE need } \\
\text { to optimize jointly }\end{array}$ & $\begin{array}{l}\text { Indoor } \mathrm{THz} \\
\text { communications }\end{array}$ & Improved the sum-rate \\
\hline$[40]$ & MISO & $\begin{array}{l}\text { Suboptimal search scheme with graphene } \\
\text { IRS and ray tracing technique }\end{array}$ & $\begin{array}{l}\text { Indoor } \mathrm{THz} \\
\text { communications }\end{array}$ & $\begin{array}{l}\text { Improved the sum-rate with reduced } \\
\text { complexity }\end{array}$ \\
\hline [41] & MIMO & $\begin{array}{l}\text { Optimization of UAV's path, IRS phase } \\
\text { shift, sub bands of THz with power } \\
\text { control }\end{array}$ & $\begin{array}{l}\text { UAV-supported } \mathrm{THz} \\
\text { communications }\end{array}$ & Improved the sum-rate \\
\hline [42] & $\begin{array}{l}\text { MIMO- } \\
\text { OFDM }\end{array}$ & $\begin{array}{l}\text { Hybrid beamforming and reflection } \\
\text { matrix are joint optimized }\end{array}$ & THz communications & $\begin{array}{l}\text { Impact of channel estimation error on data } \\
\text { rate with imperfect CSI and maximized sum } \\
\text { rate with perfect CSI }\end{array}$ \\
\hline [43] & $\begin{array}{l}\text { Ultra- } \\
\text { Massive } \\
\text { MIMO }\end{array}$ & Deployment of plasmonic arrays & $\begin{array}{l}\text { Mm wave and } \mathrm{THz} \\
\text { communications }\end{array}$ & $\begin{array}{l}\text { Significant improvements } \\
\text { communication distance and data rate }\end{array}$ \\
\hline
\end{tabular}




\subsection{Spectral efficiency optimization in IRS communication at THz}

In this section, we review recent study on spectral efficiency optimization in IRS based $\mathrm{THz}$ communications. The spectral efficiency is maximized by using plasmonic antenna array and jointly optimized the beam forming beam at transceivers in UM-MIMO and achieved a desired power level [44]. Chen et al. [45] has studied the application of the IRS for improving the spectral efficiency in $\mathrm{THz}$ communication system. Authors have utilized the discrete phase-shifts at IRS and precoder at base station for THz MIMO communication system. The non-convex optimization issue is resolved by invoking cross entropy algorithm for optimal phase shift matrix and water filing solution to enhance the precoder at BS. A descent method for MIMO is proposed recently [46] to enhance spectral efficiency with negligible complexity compared to Cross entropy-based method. Also the work presented [30] for channel estimation method also achieves a good spectral efficiency for THz massive MIMO IRS-assisted system. Table 5 focus on review on recent study on spectral efficienty using IRS in terahertz band.

Table 5. Spectral efficiency via IRS in THz

\begin{tabular}{cclll}
\hline Ref & Scheme & \multicolumn{1}{c}{ Design methodology } & Application scenario & \multicolumn{1}{c}{ Justification } \\
\hline [44] & $\begin{array}{c}\text { Ultra- } \\
\text { Massive } \\
\text { MIMO }\end{array}$ & $\begin{array}{l}\text { Joint beamforming with plasmonic antenna } \\
\text { arrays enabled by graphene meta surface }\end{array}$ & $\begin{array}{l}\text { Mm Wave and THz } \\
\text { communications }\end{array}$ & Improved spectral efficiency \\
[45] & MIMO & $\begin{array}{l}\text { Phase-shifts matrix and the precoder matrix } \\
\text { of IRS } \\
\text { Taylor expansion aided gradient descent } \\
\text { (TE-GD) scheme }\end{array}$ & $\begin{array}{l}\text { THz } \\
\text { communications } \\
\text { THz } \\
\text { communications }\end{array}$ & $\begin{array}{l}\text { Improved spectral efficiency } \\
\text { low computational complexity }\end{array}$ \\
\hline
\end{tabular}

\subsection{Meta Material's and metasurface based design for IRS Aided THz Communication}

Apart from $\mathrm{THz}$ communication system, numerous material properties support THz-band IRS systems. Dash et al. [27], a graphene meta surface at low $\mathrm{THz}$ frequencies with a simple structure has been developed. Authors have analyzed the performance under normal and oblique incidences in TE and TM mode polarization. The modeling and design of 3D curvilinear metasurface-based structures for cloaking application and for THz communication is developed [47]. Yang et al. [47] reviewed the terahertz beam steering method from conventional to reconfigurable metasurface routes based on various materials. The use of beam steering in terahertz plasmonic antennas with digital metasurface based on graphene is proposed [48]. Table 6 shows review on metamaterial and metasurface based design for tera herts communication.

Table 6. Meta material's and metasurface based design for IRS Aided THz communication

\begin{tabular}{|c|c|c|c|c|}
\hline Ref & Scheme & Design methodology & Application scenario & Justification \\
\hline [27] & $\begin{array}{c}\mathrm{THz} \\
\text { Communication }\end{array}$ & Graphene meta surface & Thz communication & $\begin{array}{l}\text { excellent absorption, zero reflection and } \\
\text { transmission at } 2.5 \mathrm{THz}\end{array}$ \\
\hline [47] & $\begin{array}{c}\mathrm{THz} \\
\text { Communication }\end{array}$ & $\begin{array}{l}\text { 3D curvilinear } \\
\text { metasurface }\end{array}$ & $\begin{array}{l}\text { Cloaking and } \mathrm{THz} \\
\text { communication }\end{array}$ & $\begin{array}{l}\text { For immidiate control of amplitude and phase } \\
\text { of the wave the link is crated between filed } \\
\text { and circuit theory }\end{array}$ \\
\hline [47] & $\begin{array}{c}\mathrm{THz} \\
\text { Communication }\end{array}$ & $\begin{array}{l}\text { Reconfigurable } \\
\text { metasurface }\end{array}$ & THz communication & $\begin{array}{l}\text { flexible for producing orbit angular } \\
\text { momentum and polarization conversion }\end{array}$ \\
\hline [48] & $\begin{array}{c}\mathrm{THz} \\
\text { Communication }\end{array}$ & $\begin{array}{l}\text { Plasmonic antennas with } \\
\text { digital metasurface }\end{array}$ & THz communication & Beam steering in all practical directions \\
\hline [43] & $\begin{array}{l}\text { Ultra-Massive } \\
\text { MIMO }\end{array}$ & $\begin{array}{l}\text { Install plasmonic arrays } \\
\text { at the transceivers }\end{array}$ & $\begin{array}{l}\text { Mm wave and } \mathrm{THz} \\
\text { communications }\end{array}$ & $\begin{array}{l}\text { Major enchancement in communication } \\
\text { distance and data rate }\end{array}$ \\
\hline
\end{tabular}

\section{DISCUSSION ON FUTURE SCOPE}

In this section, the challeneghs and results are discussed based on the algorithms applied in order to receive the probable solutions. The techniques used for the intelligent reflecting surfaces assisted $\mathrm{THz}$ communication in analyzed in brief and it enhance the communication quality. Also, in this section we highlight a possible research direction in $\mathrm{THz}$ communication for future investigation that enables the deployment of $\mathrm{THz}$ links and opens the door in the direction of various applications such as mobile heterogeneous networks (HetNtes), machine learing, medium access protocol and artificial intelligence.

\subsection{Terahertz communications for mobile HetNets}

High path loss and requirements of greatly directional antennas are the main drawback of $\mathrm{THz}$ communication systems. By using the femtocell regime these could turn into favorable attributes. Femtocells are able to redue the distance between serving base-station and the user and though maintain a high SNR at the 
receiver they also enhance the frequency reuse capacity of the THz systems. These femtocells are also known as low-power nodes, and can be used in home service, shopping malls and in various other applications. These have led to a new era of $\mathrm{THz}$ communications for mobile heterogeneous networks (HetNets) to improve the data rate at the level of Tbps [49].

\subsection{Compressive sensing and machine learning (ML)}

To enahce the $\mathrm{THz}$ communicaiton, compressive sensing and machine learning techniques are promising. To enhace the femtocell, machine learning could be aso used as articifalc intelligence tool to support the smart radio terminals. ML methods can also address the number of nano-scale biomedical challenges, including once that refer to molecular and nano-scale $\mathrm{THz}$ communications.

\subsection{Medium access protocol for $\mathrm{THz}$ communication}

There are many challenges on $\mathrm{THz}$ medium access control (MAC) design modeled by the $\mathrm{THz}$ communications like, deafness problem, requirement for the coverage of transmission and the demand of deafness avoidance, cell boundary etc. These challenges are addressed in the literature which could be use with physical layer signal processing schemes. MAC protocals technology is supported by the tertrahertz communication with the unique feature of tetrahertz band as scattering, high path loss and reflection pose new challenges and results in short communication [50]. Pure and hybrid plasmonic tetrahertz antennas provide the new degree of freedom to design the graphene-based antennas for tetrahertz band operation [51].

\subsection{Artifical intelligence (AI)}

The presence of interconnected devices with the accessibility of data has permitted the successful integration of $\mathrm{AI}$ in wireless communication. 6G shall appear as an intelligent information system which is driven by the current AI technologies. Also, the shifting of things to connected intelligence with high rate and low latency control requires the usage of $\mathrm{THz}$ frequeney band.

\section{CONCLUSION}

A broad survey on IRS based THz communication system is presented. The use of IRS in wireless communication has set up new shift in the design of wireless system. Firstly, we have presented an overview of the terahertz band and their limitations. Then we have focused on how IRS is useful to overcome these limitations and role of IRS in terahertz. Even though reserch on IRS-based system in THz is still in its early stages, this paper categorizes the role of IRS in various domain of THz like channel modeling, data and spectral efficiency improvement, antenna designing and secrecy rate along with the directions for further investigation. We can say that upcoming years will have more study for the IRS aided THz communication to shape next generation communications.

\section{REFERENCES}

[1] Z. Chen et al., "A survey on terahertz communications," China Communications, vol. 16, no. 2, pp. 1-35, Feb. 2019, doi: 10.12676/j.cc.2019.02.001

[2] I. F. Akyildiz, J. M. Jornet, and C. Han, "Terahertz band: Next frontier for wireless communications," Physical Communication, vol. 12, pp. 16-32, Sep. 2014, doi: 10.1016/j.phycom.2014.01.006.

[3] C. Han, Y. Wu, Z. Chen, and X. Wang, "Terahertz Communications (TeraCom): Challenges and Impact on 6G Wireless Systems," Dec. 2019, [Online]. Available: http://arxiv.org/abs/1912.06040.

[4] H. Elayan, O. Amin, B. Shihada, R. M. Shubair, and M.-S. Alouini, "Terahertz Band: The Last Piece of RF Spectrum Puzzle for Communication Systems," IEEE Open Journal of the Communications Society, vol. 1, pp. 1-32, 2019, doi: 10.1109/ojcoms.2019.2953633.

[5] P. H. Siegel, "Terahertz technology," IEEE Transactions on Microwave Theory and Techniques, vol. 50, no. 3, pp. 910-928, Mar. 2002, doi: 10.1109/22.989974

[6] J. Federici and L. Moeller, "Review of terahertz and subterahertz wireless communications," Journal of Applied Physics, vol. 107, no. 11, p. 111101, Jun. 2010, doi: 10.1063/1.3386413.

[7] T. Kleine-Ostmann and T. Nagatsuma, "A review on terahertz communications research," Journal of Infrared, Millimeter, and Terahertz Waves, vol. 32, no. 2, pp. 143-171, Jan. 2011, doi: 10.1007/s10762-010-9758-1.

[8] T. Nagatsuma et al., "Terahertz wireless communications based on photonics technologies," Optics Express, vol. 21, no. 20, p. 23736, Sep. 2013, doi: 10.1364/oe.21.023736.

[9] K. C. Huang and Z. Wang, "Terahertz terabit wireless communication," IEEE Microwave Magazine, vol. 12, no. 4, pp. 108-116, Jun. 2011, doi: 10.1109/MMM.2011.940596.

[10] T. Nagatsuma, "Terahertz technologies: Present and future,” IEICE Electronics Express, vol. 8, no. 14, pp. 1127-1142, 2011, doi: 10.1587/elex.8.1127.

[11] T. Kürner and S. Priebe, "Towards THz communications - Status in research, standardization and regulation," Journal of Infrared, Millimeter, and Terahertz Waves, vol. 35, no. 1, pp. 53-62, Aug. 2014, doi: 10.1007/s10762-013-0014-3.

[12] A. Hirata and M. Yaita, "Ultrafast Terahertz Wireless Communications Technologies," IEEE Transactions on Terahertz Science and Technology, vol. 5, no. 6, pp. 1128-1132, 2015. 
[13] K. Sengupta, T. Nagatsuma, and D. M. Mittleman, “Terahertz integrated electronic and hybrid electronic-photonic systems," Nature Electronics, vol. 1, no. 12, pp. 622-635, Dec. 2018, doi: 10.1038/s41928-018-0173-2.

[14] S. Mumtaz, J. M. Jornet, J. Aulin, W. H. Gerstacker, X. Dong, and B. Ai, "Terahertz Communication for Vehicular Networks," IEEE Transactions on Vehicular Technology, vol. 66, no. 7, pp. 5617-5625, Jul. 2017, doi: 10.1109/TVT.2017.2712878.

[15] Y. Chen, X. Cai, and C. Han, "Wave Propagation Modeling for mmWave and Terahertz Wireless Networks-on-Chip Communications," in IEEE International Conference on Communications, May 2019, vol. 2019-May, doi: 10.1109/ICC.2019.8761352.

[16] J. F. O'Hara, S. Ekin, W. Choi, and I. Song, "A Perspective on Terahertz Next-Generation Wireless Communications," Technologies, vol. 7, no. 2, p. 43, Jun. 2019, doi: 10.3390/technologies7020043.

[17] M. A. Jamshed, A. Nauman, M. A. B. Abbasi, and S. W. Kim, "Antenna Selection and Designing for THz Applications: Suitability and Performance Evaluation: A Survey," IEEE Access, vol. 8, pp. 113246-113261, 2020, doi: 10.1109/ACCESS.2020.3002989.

[18] M. H. Rahaman, A. Bandyopadhyay, S. Pal, and K. P. Ray, "Reviewing the Scope of THz Communication and a Technology Roadmap for Implementation," IETE Technical Review (Institution of Electronics and Telecommunication Engineers, India), vol. 38, no. 5, pp. 465-478, Jun. 2021, doi: 10.1080/02564602.2020.1771221.

[19] H. Sarieddeen, M. S. Alouini, and T. Y. Al-Naffouri, "An Overview of Signal Processing Techniques for Terahertz Communications," Proceedings of the IEEE, vol. 109, no. 10, pp. 1628-1665, May 2021, doi: 10.1109/JPROC.2021.3100811.

[20] H. Sarieddeen, N. Saeed, T. Y. Al-Naffouri, and M. S. Alouini, "Next generation terahertz communications: A rendezvous of sensing, imaging, and localization," IEEE Communications Magazine, vol. 58, no. 5, pp. 69-75, May 2020, doi: 10.1109/MCOM.001.1900698

[21] L. U. Khan, I. Yaqoob, M. Imran, Z. Han, and C. S. Hong, "6G Wireless Systems: A Vision, Architectural Elements, and Future Directions," IEEE Access, vol. 8, pp. 147029-147044, 2020, doi: 10.1109/ACCESS.2020.3015289.

[22] B. Ferguson and X. C. Zhang, "Materials for terahertz science and technology," Nature Materials, vol. 1, no. 1, pp. 26-33, Sep. 2002, doi: 10.1038/nmat708.

[23] H. J. Song and T. Nagatsuma, "Present and future of terahertz communications," IEEE Transactions on Terahertz Science and Technology, vol. 1, no. 1, pp. 256-263, Sep. 2011, doi: 10.1109/TTHZ.2011.2159552.

[24] S. Abadal, C. Han, and J. M. Jornet, "Wave Propagation and Channel Modeling in Chip-Scale Wireless Communications: A Survey from Millimeter-Wave to Terahertz and Optics," IEEE Access, vol. 8, pp. 278-293, 2020, doi: 10.1109/ACCESS.2019.2961849.

[25] Q. Wu, S. Zhang, B. Zheng, C. You, and R. Zhang, "Intelligent Reflecting Surface-Aided Wireless Communications: A Tutorial," IEEE Transactions on Communications, vol. 69, no. 5, pp. 3313-3351, May 2021, doi: 10.1109/TCOMM.2021.3051897.

[26] C. Pan et al., "Reconfigurable Intelligent Surfaces for 6G Systems: Principles, Applications, and Research Directions," IEEE Communications Magazine, vol. 59, no. 6, pp. 14-20, Nov. 2021, doi: 10.1109/MCOM.001.2001076.

[27] S. Dash, C. Liaskos, I. F. Akyildiz, and A. Pitsillides, "Wideband Perfect Absorption Polarization Insensitive Reconfigurable Graphene Metasurface for THz Wireless Environment," in Proceedings of 2019 IEEE Microwave Theory and Techniques in Wireless Communications, MTTW 2019, Oct. 2019, pp. 93-96, doi: 10.1109/MTTW.2019.8897231.

[28] P. Varzakas, "Channel capacity per user in a power and rate adaptive hybrid DS/FFH-CDMA cellular system over Rayleigh fading channels," International Journal of Communication Systems, vol. 25, no. 7, pp. 943-952, Jun. 2012, doi: 10.1002/dac.1298

[29] P. Varzakas, "Average channel capacity for Rayleigh fading spread spectrum MIMO systems," International Journal of Communication Systems, vol. 19, no. 10, pp. 1081-1087, 2006, doi: 10.1002/dac.784.

[30] R. A. Koutsiamanis, G. Z. Papadopoulos, T. L. Jenschke, P. Thubert, and N. Montavont, "Meet the PAREO Functions: Towards Reliable and Available Wireless Networks," in IEEE International Conference on Communications, Jun. 2020, vol. 2020-June, doi: 10.1109/ICC40277.2020.9149206.

[31] X. Ma, Z. Chen, Y. Chi, W. Chen, L. Du, and Z. Li, "Channel estimation for intelligent reflecting surface enabled terahertz MIMO systems,” Jun. 2020, doi: 10.1109/ICCWorkshops49005.2020.9145343.

[32] Z. Wang, L. Liu, and S. Cui, "Channel Estimation for Intelligent Reflecting Surface Assisted Multiuser Communications: Framework, Algorithms, and Analysis," IEEE Transactions on Wireless Communications, vol. 19, no. 10, pp. 6607-6620, Oct. 2020, doi: 10.1109/TWC.2020.3004330.

[33] B. Ning, Z. Chen, W. Chen, Y. Du, and J. Fang, “Terahertz Multi-User Massive MIMO with Intelligent Reflecting Surface: Beam Training and Hybrid Beamforming," IEEE Transactions on Vehicular Technology, vol. 70, no. 2, pp. 1376-1393, Feb. 2021, doi: 10.1109/TVT.2021.3052074

[34] X. Ma et al., "Joint Channel Estimation and Data Rate Maximization for Intelligent Reflecting Surface Assisted Terahertz MIMO Communication Systems," IEEE Access, vol. 8, pp. 99565-99581, 2020, doi: 10.1109/ACCESS.2020.2994100.

[35] J. Qiao and M. S. Alouini, "Secure transmission for intelligent reflecting surface-assisted mmWave and terahertz systems," IEEE Wireless Communications Letters, vol. 9, no. 10, pp. 1743-1747, Oct. 2020, doi: 10.1109/LWC.2020.3003400.

[36] B. Ning, Z. Chen, W. Chen, and L. Li, "Improving security of THz communication with intelligent reflecting surface," Dec. 2019, doi: 10.1109/GCWkshps45667.2019.9024636.

[37] W. Chen, Z. Chen, X. Ma, Y. Chi, and Z. Li, "Secrecy rate optimization for intelligent reflecting surface aided multi-input-singleoutput terahertz communication," Microwave and Optical Technology Letters, vol. 62, no. 8, pp. 2760-2765, Mar. 2020, doi: 10.1002/mop.32373.

[38] H. Shen, W. Xu, S. Gong, Z. He, and C. Zhao, "Secrecy Rate Maximization for Intelligent Reflecting Surface Assisted MultiAntenna Communications," IEEE Communications Letters, vol. 23, no. 9, pp. 1488-1492, Sep. 2019, doi: 10.1109/LCOMM.2019.2924214.

[39] Y. Pan, K. Wang, C. Pan, H. Zhu, and J. Wang, "Sum Rate Maximization for Intelligent Reflecting Surface Assisted Terahertz Communications," Aug. 2020, [Online]. Available: http://arxiv.org/abs/2008.12246.

[40] X. Ma et al., "Intelligent reflecting surface enhanced indoor terahertz communication systems," Nano Communication Networks, vol. 24, p. 100284, May 2020, doi: 10.1016/j.nancom.2020.100284.

[41] Y. Pan, K. Wang, C. Pan, H. Zhu, and J. Wang, "UAV-Assisted and Intelligent Reflecting Surfaces-Supported Terahertz Communications," IEEE Wireless Communications Letters, vol. 10, no. 6, pp. 1256-1260, Jun. 2021, doi: 10.1109/LWC.2021.3063365.

[42] W. Hao et al., "Robust Design for Intelligent Reflecting Surface-Assisted MIMO-OFDMA Terahertz IoT Networks," IEEE Internet of Things Journal, vol. 8, no. 16, pp. 13052-13064, Sep. 2021, doi: 10.1109/JIOT.2021.3064069.

[43] S. Nie, J. M. Jornet, and I. F. Akyildiz, "Intelligent Environments Based on Ultra-massive Mimo Platforms for Wireless Communication in Millimeter Wave and Terahertz Bands," in ICASSP, IEEE International Conference on Acoustics, Speech and Signal Processing - Proceedings, May 2019, vol. 2019-May, pp. 7849-7853, doi: 10.1109/ICASSP.2019.8683394. 
[44] S. Nie and I. F. Akyildiz, "Beamforming in Intelligent Environments based on Ultra-Massive MIMO Platforms in Millimeter Wave and Terahertz Bands," in ICASSP, IEEE International Conference on Acoustics, Speech and Signal Processing - Proceedings, May 2020, vol. 2020-May, pp. 8683-8687, doi: 10.1109/ICASSP40776.2020.9053786.

[45] W. Chen, Z. Chen, X. Ma, Y. Chi, and Z. Li, "Spectral efficiency optimization for intelligent reflecting surface aided multi-input multi-output terahertz system," Microwave and Optical Technology Letters, vol. 62, no. 8, pp. 2754-2759, Mar. 2020, doi: 10.1002/mop.32362.

[46] Z. Chen, W. Chen, X. Ma, Z. Li, Y. Chi, and C. Han, "Taylor Expansion Aided Gradient Descent Schemes for IRS-Enabled Terahertz MIMO Systems,” Apr. 2020, doi: 10.1109/WCNCW48565.2020.9124799.

[47] X. Fu, F. Yang, C. Liu, X. Wu, and T. J. Cui, "Terahertz Beam Steering Technologies: From Phased Arrays to Field-Programmable Metasurfaces," Advanced Optical Materials, vol. 8, no. 3, p. 1900628, Aug. 2020, doi: 10.1002/adom.201900628.

[48] S. E. Hosseininejad, K. Rouhi, M. Neshat, A. Cabellos-Aparicio, S. Abadal, and E. Alarcon, "Digital metasurface based on graphene: An application to beam steering in terahertz plasmonic antennas," IEEE Transactions on Nanotechnology, vol. 18, pp. 734-746, 2019, doi: 10.1109/TNANO.2019.2923727.

[49] K. Tekbiyik, A. R. Ekti, G. K. Kurt, A. Gorcin, and H. Yanikomeroglu, "A Holistic Investigation of Terahertz Propagation and Channel Modeling toward Vertical Heterogeneous Networks," IEEE Communications Magazine, vol. 58, no. 11, pp. 14-20, Nov. 2020, doi: 10.1109/MCOM.001.2000302.

[50] S. Ghafoor, N. Boujnah, M. H. Rehmani, and A. Davy, "MAC Protocols for Terahertz Communication: A Comprehensive Survey," IEEE Communications Surveys and Tutorials, vol. 22, no. 4, pp. 2236-2282, 2020, doi: 10.1109/COMST.2020.3017393.

[51] X. W. Yao and J. M. Jornet, "TAB-MAC: Assisted beamforming MAC protocol for Terahertz communication networks," Nano Communication Networks, vol. 9, pp. 36-42, Sep. 2016, doi: 10.1016/j.nancom.2016.07.003.

\section{BIOGRAPHIES OF AUTHORS}

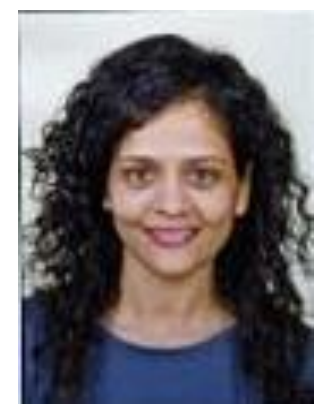

Arpita Patel (D) 8D SC P is Associate Professor at Charotar University of Science and Technolgy, Changa, Gujarat, India. She Holds a PhD degree in Electronics and Communication Enginnering with specialization in Wirelss communication. Her research areas are signal processing, multiple access schemes for $6 \mathrm{G}$ communication and MIMO communication. She can be contacted at email: arpitapatel.ec@ charusat.ac.in.

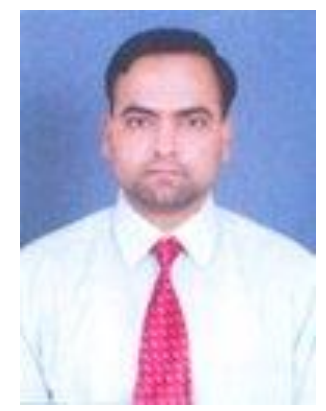

Aasheesh Shukla (D) $8 \mathrm{SC}$ S is Associate Dean Academic Affairs, Member in Internal Quality Assurance cell (IQAC), GLA University, Mathura. He Holds a PhD degree in Electronics and Communication Enginnering with specialization in Wirelss communication. His research areas are multiple access schemes for $6 \mathrm{G}$ communication, MIMO Massive Access Communication, and signal processing. He can be contacted at email: aasheesh.shukla@gla.ac.in. 